PROCEEDINGS OF THE

AMERICAN MATHEMATICAL SOCIETY

Volume 138, Number 4, April 2010, Pages 1501-1510

S 0002-9939(09)10143-0

Article electronically published on November 23, 2009

\title{
BOCKSTEIN THEOREM FOR NILPOTENT GROUPS
}

\author{
M. CENCELJ, J. DYDAK, A. MITRA, AND A. VAVPETIČ \\ (Communicated by Alexander N. Dranishnikov)
}

\begin{abstract}
We extend the definition of Bockstein basis $\sigma(G)$ to nilpotent groups $G$. A metrizable space $X$ is called a Bockstein space if $\operatorname{dim}_{G}(X)=$ $\sup \left\{\operatorname{dim}_{H}(X) \mid H \in \sigma(G)\right\}$ for all Abelian groups $G$. The Bockstein First Theorem says that all compact spaces are Bockstein spaces.

Here are the main results of the paper:

Theorem 0.1. Let $X$ be a Bockstein space. If $G$ is nilpotent, then $\operatorname{dim}_{G}(X) \leq$ 1 if and only if $\sup \left\{\operatorname{dim}_{H}(X) \mid H \in \sigma(G)\right\} \leq 1$.

Theorem 0.2. $X$ is a Bockstein space if and only if $\operatorname{dim}_{\mathbf{Z}_{(l)}}(X)=\operatorname{dim}_{\hat{Z}_{(l)}}(X)$

for all subsets $l$ of prime numbers.
\end{abstract}

\section{INTRODUCTION}

We use the Kuratowski notation $X \tau M$ in the case when every map from a closed subset of $X$ to $M$ can be extended over all $X$.

Recall that the cohomological dimension of a space $X$ with respect to an Abelian group $G$ is less than or equal to $n$, denoted by $\operatorname{dim}_{G} X \leq n$, if $\tilde{H}^{n+1}(X, A ; G)=0$ for all closed $A \subset X$.

Of basic importance in cohomological dimension theory is the Bockstein basis $\sigma(G)$ of an Abelian group $G$ (see Definition 3.1) and the following result of Bockstein (see [12] or 4]):

Theorem 1.1 (Bockstein First Theorem). If $X$ is a compact space, then

$$
\operatorname{dim}_{G}(X)=\sup \left\{\operatorname{dim}_{H}(X) \mid H \in \sigma(G)\right\} .
$$

The aim of this paper is to generalize Theorem 1.1 to nilpotent groups. There are two issues to resolve first:

(1) Define cohomological dimension with respect to non-Abelian groups.

(2) Define the Bockstein basis of nilpotent groups.

The definition of $\operatorname{dim}_{G}(X)$ for $G$ non-Abelian was first introduced by A. Dranishnikov and D. Repovš [5] as follows: By [4, Theorem 1.1], $\operatorname{dim}_{G}(X) \leq n$ is equivalent (for Abelian groups $G$ ) to $X \tau K(G, n)$, where $K(G, n)$ is an Eilenberg-MacLane

Received by the editors September 23, 2008, and, in revised form, April 21, 2009.

2010 Mathematics Subject Classification. Primary 54F45; Secondary 55M10, 54C20.

Key words and phrases. Extension dimension, cohomological dimension, absolute extensor, nilpotent groups.

This work supported in part by the Slovenian-USA research grant BI-US/05-06/002 and the ARRS grants P1-0292-0101 and J1-2057-0101.

The second-named author was partially supported by MEC, MTM2006-0825.

(C)2009 American Mathematical Society 
space. One ought to use the same equivalence in the definition of $\operatorname{dim}_{G}(X)$ for non-Abelian groups. For nilpotent groups $G$ this definition was used for the characterization of nilpotent $\mathrm{CW}$ complexes as absolute extensors of metric compacta (see [3]). There is no Eilenberg-MacLane space $K(G, n), 1<n<\infty$, for nonAbelian groups $G$, so $\operatorname{dim}_{G} X \in\{0,1, \infty\}$. Since $\operatorname{dim}_{G} X=0$ means $\operatorname{dim}(X)=0$ (see Lemma 1.2), the only interesting question is if $X \tau K(G, 1)$ holds.

Our definition of the Bockstein basis for nilpotent groups can be found in Definition 3.2

The remainder of this section is devoted to elementary properties of cohomological dimension over non-Abelian groups.

Lemma 1.2. If $X$ is a metrizable space and $\operatorname{dim}_{G}(X)=0$ for some group $G \neq 1$, then $\operatorname{dim}_{H}(X)=0$ for any group $H$.

Proof. We will show that for a nontrivial group $G$ we have $\operatorname{dim}_{G}(X)=0$ if and only if $\operatorname{dim}_{\mathbf{Z}_{2}}(X)=0$ and $\operatorname{dim}_{\mathbf{Z}_{2}}(X)=0$ means $\operatorname{dim}(X)=0$..

Suppose $\operatorname{dim}_{G}(X)=0$ for some $G \neq 1$. Let $i: \mathbf{Z}_{2} \rightarrow G$ be an injection and let $r: G \rightarrow \mathbf{Z}_{2}$ be a map such that $r \circ i=i d_{\mathbf{Z}_{2}}$. Let $A$ be a closed subset of $X$ and $f: A \rightarrow K\left(\mathbf{Z}_{2}, 0\right)=\mathbf{Z}_{2}$ a map. Then there exists an extension $f^{\prime}: X \rightarrow G$ of $i \circ f: A \rightarrow G$. Then $F=r \circ f^{\prime}: X \rightarrow \mathbf{Z}_{2}$ is an extension of $f$.

Suppose that $\operatorname{dim}_{\mathbf{Z}_{2}}(X)=0$. Let $A \subset X$ be a closed subset and let $f: A \rightarrow G$ be a continuous map. For every $g \in G$ we define $A_{g}=f^{-1}(g)$ and $B_{g}=\{x \in X \mid$ $\left.d\left(x, A \backslash A_{g}\right) \leq d\left(x, A_{g}\right)\right\}$. Because $A \backslash A_{g}$ is a closed subset of $X, B_{g}$ is a closed subset of $X$. Because $B_{g} \cap A_{g}=\emptyset$ we can define a continuous map $f_{g}: A_{g} \cup B_{g} \rightarrow \mathbf{Z}_{2}$ by $f_{g}\left(A_{g}\right)=1$ and $f_{g}\left(B_{g}\right)=0$. Let $F_{g}: X \rightarrow \mathbf{Z}_{2}$ be an extension of $f_{g}$. For every $g \in G$ we define $X_{g}=F_{g}^{-1}(1)$. Sets $X_{g}$ are open and closed in $X$ and pairwise disjoint. So we can define a continuous map $F: X \rightarrow G$ as

$$
F(x)= \begin{cases}g & ; x \in X_{g}, \\ e & ; x \notin \bigcup_{g \in G} X_{g} .\end{cases}
$$

The map $F$ is an extension of $f$.

Lemma 1.3. Let $X$ be a metrizable space. If $1 \rightarrow J \rightarrow G \rightarrow I \rightarrow 1$ is an exact sequence of groups and $\operatorname{dim}_{J} X \leq 1$, then $\operatorname{dim}_{I}(X) \leq 1$ if and only if $\operatorname{dim}_{G}(X) \leq 1$.

Proof. In view of Lemma 1.2 the only interesting case is that of $\operatorname{dim}_{J} X=1$. Use the fibration $K(J, 1) \rightarrow K(G, 1) \rightarrow K(I, 1)$ and the fact that $X \tau K(J, 1)$ to conclude $X \tau K(I, 1)$ if and only if $X \tau K(G, 1)$.

If $G$ is a group, then $\operatorname{Ab}(G)$ is its abelianization.

Lemma 1.4. If $X$ is a metrizable space, then $\operatorname{dim}_{A b(G)}(X) \leq \operatorname{dim}_{G} X$ for any group $G$.

Proof. In view of Lemma 1.2 the only interesting case is that of $G$ non-Abelian and $\operatorname{dim}_{G} X=1$. Since $X \tau K(G, 1)$ one gets $X \tau K\left(H_{1}(K(G, 1)), 1\right)$ by Theorem 3.4 of 8]. As $H_{1}(K(G, 1))=\operatorname{Ab}(G)$, we are done.

\section{Nilpotent Groups}

If $A, B \subset G$ are subgroups, then the commutator subgroup $[A, B]$ is a group generated by all commutators $[a, b], a \in A$ and $b \in B$. The lower central series $\left\{\Gamma_{n}(G)\right\}$ for a group $G$ is defined as follows: $\Gamma_{1}(G)=G$, and $\Gamma_{n+1}(G)=\left[\Gamma_{n}(G), G\right]$. 
If a group $G$ is nilpotent, then there exists an integer $c$ such that $\Gamma_{c}(G) \neq\{1\}$ but $\Gamma_{c+1}(G)=\{1\}$. The number $c$ (denoted by $h(G)$ ) is called the nilpotency class of the nilpotent group $G$ or its Hirsch length. Abelian groups are nilpotent of Hirsch length 1. By [14, Theorem 3.1], for every $n$ there exists an epimorphism

$$
\otimes^{n} \mathrm{Ab}(G) \rightarrow \Gamma_{n}(G) / \Gamma_{n+1}(G) .
$$

In particular, there is an epimorphism $\otimes^{c} \mathrm{Ab}(G) \rightarrow \Gamma_{c}(G)$. It follows from the definition that $\Gamma_{c}(G)$ is in the center of $G$. Therefore $1 \rightarrow \Gamma_{c}(G) \rightarrow G \rightarrow G / \Gamma_{c}(G) \rightarrow 1$ is a central extension. A short calculation shows that the epimorphism $G \rightarrow$ $G / \Gamma_{c}(G)$ induces the trivial homomorphism $\Gamma_{c}(G) \rightarrow \Gamma_{c}\left(G / \Gamma_{c}(G)\right)=1$. Therefore $G / \Gamma_{c}(G)$ is nilpotent of nilpotency class strictly less than $c$. This motivates the following definition.

Definition 2.1. A central extension $K \rightarrow G \rightarrow I$ of groups where $G$ is nilpotent (or equivalently $I$ is nilpotent), for which there exists an epimorphism $\otimes^{n} \mathrm{Ab}(G) \rightarrow K$ for some $n$, is called a nilpotent central extension.

Thus, for every (non-Abelian) nilpotent group $G$, there exists a nilpotent central extension $K \rightarrow G \rightarrow I$ such that the Hirsch length of $I$ is less than the Hirsch length of $G$.

Lemma 2.2. Let $1 \rightarrow K \rightarrow G \stackrel{\pi}{\rightarrow} I \rightarrow 1$ be a central extension of nilpotent groups.

(a) If $K$ and $I$ are $p$-divisible, then $G$ is p-divisible.

(b) If the extension is a nilpotent central extension and $G$ is p-divisible, then $K$ and $I$ are $p$-divisible.

Proof. Suppose $K$ and $I$ are $p$-divisible. Let $g \in G$. Then $\pi(g)=i^{p}$ for some $i \in I$. Let $\bar{g} \in G$ be such that $\pi(\bar{g})=i$. Then $\pi\left(\bar{g}^{p} g^{-1}\right)=1$, so $\bar{g}^{p} g^{-1}=k^{p}$ for some $k \in K$. Because $k \in K \subset C(G), g=\left(\bar{g} k^{-1}\right)^{p}$, so $G$ is $p$-divisible.

If $G$ is $p$-divisible, then any epimorphic image of $G$ is $p$-divisible. Thus both $I$ and $\operatorname{Ab}(G)$ are $p$-divisible. As there is an epimorphism $\otimes^{n} \mathrm{Ab}(G) \rightarrow K, K$ is also $p$-divisible.

Lemma 2.3. Suppose $\mathcal{P}_{i}, i=1,2$, are two classes of nilpotent groups such that for any nilpotent central extension $1 \rightarrow K \rightarrow G \rightarrow I \rightarrow 1$ where $h(I)<h(G)$ the following conditions hold:

(a) $K$ and $I$ belong to $\mathcal{P}_{1}$ if $G \in \mathcal{P}_{1}$,

(b) $G \in \mathcal{P}_{2}$ if $K, I \in \mathcal{P}_{2}$.

If $A \in \mathcal{P}_{1} \Longrightarrow A \in \mathcal{P}_{2}$ for all Abelian groups $A$, then $\mathcal{P}_{1} \subset \mathcal{P}_{2}$.

Proof. We prove the implication $G \in \mathcal{P}_{1} \Longrightarrow G \in \mathcal{P}_{2}$ by induction on the Hirsch length of $G$. By assumption the implication holds for an Abelian group $G$. Therefore suppose $1 \rightarrow K \rightarrow G \rightarrow I \rightarrow 1$ is a nilpotent central extension of groups and $I$ is of lower Hirsch length than the Hirsch length of $G$. By (a) we have $K, I \in \mathcal{P}_{1}$. By the inductive hypothesis, $K, I \in \mathcal{P}_{2}$. The condition (b) yields $G \in \mathcal{P}_{2}$.

Corollary 2.4. Let $A$ be an Abelian group and $n \in\{1,2\}$. Consider the following statements:

(1) $\widetilde{H}_{*}(G ; A)=0$,

(2) $\widetilde{H}_{i}(G ; A)=0$ for all $i \leq n$.

If (1) is equivalent to (2) for all Abelian groups $G$, then the two statements are equivalent for all nilpotent groups $G$. 
Proof. Let $\mathcal{P}_{1}=\mathcal{P}_{1}^{r}$ (respectively, $\mathcal{P}_{2}=\mathcal{P}_{2}^{r}$ ) be the class of all nilpotent groups $G$ such that $\widetilde{H}_{i}(G ; A)=0$ for all $i \leq n$ (respectively, $\widetilde{H}_{i}(G ; A)=0$ for all $i$ ) and $h(G) \leq r$. Our goal is to prove, by induction on $r$, that $\mathcal{P}_{1}^{r}=\mathcal{P}_{2}^{r}$. It is clearly so for $r=1$. Assume $\mathcal{P}_{1}^{m}=\mathcal{P}_{2}^{m}$ for all $m<r$.

Suppose $1 \rightarrow K \rightarrow G \rightarrow I \rightarrow 1$ is a nilpotent central extension of groups such that $h(I)<h(G)=r$. If $G \in \mathcal{P}_{j}^{r}$, then $H_{1}(K ; A)=0$, which implies $H_{i}(G ; A) \rightarrow H_{i}(I ; A)$ is an epimorphism for $i \leq 2$, so $H_{i}(I ; A)=0$ for $1 \leq i \leq n$. By the inductive assumption, $H_{i}(I ; A)=0$ for all $i \geq 1$. If $\widetilde{H}_{i}(K ; A)$ is the first nontrivial reduced homology group of $K$, then the Leray-Serre spectral sequence implies $H_{i+1}(I ; A) \neq 0$, a contradiction. Thus both $K$ and $I$ belong to $\mathcal{P}_{j}^{r}$. Conversely, if $K, I \in \mathcal{P}_{j}^{r-1}$, then (by the inductive assumption) they have trivial homology with coefficients in $A$ resulting in $G$ having trivial homology with coefficients in $A$ and $G \in \mathcal{P}_{j}^{r}$. Applying Lemma 2.3 one gets $\mathcal{P}_{1}^{r}=\mathcal{P}_{2}^{r}$.

\section{BOCKSTEIN BASIS}

If $G$ is a group, then $\operatorname{Tor}(G)$ is the subgroup generated by torsion elements of $G$, $\operatorname{Tor}_{p}(G)$ is the subgroup generated by all elements of $G$ whose order is a power of $p, F_{p}(G)=G / \operatorname{Tor}_{p}(G)$, and $F(G)=G / \operatorname{Tor}(G)$.

The Bockstein groups are: rationals $\mathbf{Q}$, cyclic groups $\mathbf{Z} / p$ of $p$ elements, $p$-adic circles $\mathbf{Z} / p^{\infty}$, and $p$-localizations of integers $\mathbf{Z}_{(p)}=\left\{\frac{m}{n} \in \mathbf{Q} \mid n\right.$ is not divisible by $\left.p\right\}$, where $p$ is a prime number. Here is a classical definition of the maximal Bockstein basis of an Abelian group $G$ :

Definition 3.1. $\sigma(G)$ is a subset of all Bockstein groups satisfying the following conditions:

(1) $\mathbf{Q} \in \sigma(G)$ if and only if $F(G) \neq 1$.

(2) $\mathbf{Z} / p^{\infty} \in \sigma(G)$ if and only if $\operatorname{Tor}_{p}(G) \neq 1$ or $F(G)$ is not divisible by $p$.

(3) $\mathbf{Z} / p \in \sigma(G)$ if and only if $\operatorname{Tor}_{p}(G)$ is not divisible by $p$ or $F(G)$ is not divisible by $p$.

(4) $\mathbf{Z}_{(p)} \in \sigma(G)$ if and only if $F(G)$ is not divisible by $p$.

Notice that our Definition 3.1 differs from that in 4 in the sense that ours is maximal (if $\mathbf{Z} / p \in \sigma(G)$, then $\mathbf{Z} / p^{\infty} \in \sigma(G)$ and if $\mathbf{Z}_{(p)} \in \sigma(G)$, then $\mathbf{Z} / p \in$ $\sigma(G)$ ) and the one in [4] is minimal (if $\mathbf{Z} / p \in \sigma(G)$ or $\mathbf{Z}_{(p)} \in \sigma(G)$, then $\mathbf{Z} / p^{\infty} \notin$ $\sigma(G))$. From the point of view of the First Bockstein Theorem, both definitions are equivalent.

Here is a definition for nilpotent groups which is more convenient in this paper as it allows using localization of short exact sequences of nilpotent groups. Recall that a (multiplicative) group is $\bar{p}$-local iff the map $x \mapsto x^{p}$ is a bijection. We call a nilpotent group $p$-local iff it is $\bar{q}$-local for all primes $q \neq p$.

Definition 3.2. Let $G$ be a nilpotent group. Then the Bockstein basis $\sigma(G)$ is defined as follows:

(1) $\mathbf{Q} \notin \sigma(G)$ if and only if $G=\operatorname{Tor}(G)$.

(2) $\mathbf{Z} / p^{\infty} \notin \sigma(G)$ if and only if $G$ is $\bar{p}$-local.

(3) $\mathbf{Z} / p \notin \sigma(G)$ if and only if $G$ is divisible by $p$.

(4) $\mathbf{Z}_{(p)} \notin \sigma(G)$ if and only if $F(G)$ is $\bar{p}$-local.

Remark 3.3. Note that according to the above definition we have

$$
\mathbf{Z}_{(p)} \in \sigma(G) \Rightarrow \mathbf{Z} / p \in \sigma(G) \Rightarrow \mathbf{Z} / p^{\infty} \in \sigma(G) .
$$


Corollary 3.4. For a nilpotent group $G$ the following statements are equivalent:

(1) $\mathbf{Q} \notin \sigma(G)$,

(2) $\widetilde{H}_{*}(G ; \mathbf{Q})=0$, and

(3) $H_{1}(G ; \mathbf{Q})=0$.

Proof. For Abelian groups, (2) and (3) are equivalent 1, Theorem V.6.4(ii)]. With Corollary 2.4 we extend this equivalence to all nilpotent groups.

Let $\mathcal{P}_{1}$ be the class of torsion nilpotent groups and let $\mathcal{P}_{2}$ be the class of nilpotent groups such that $H_{1}(G ; \mathbf{Q})=0$ (i.e., $\mathrm{Ab}(G)$ is torsion). Use Lemma 2.3 to conclude that $\mathcal{P}_{1}=\mathcal{P}_{2}$ and thus (1) and (3) are equivalent.

Corollary 3.5. For a nilpotent group $G, \mathbf{Z} / p \notin \sigma(G)$ if and only if $H_{1}(G ; \mathbf{Z} / p)=0$.

Proof. Let $\mathcal{P}_{1}$ be the class of $p$-divisible nilpotent groups and let $\mathcal{P}_{2}$ be the class of nilpotent groups such that $H_{1}(G ; \mathbf{Z} / p)=0$. Use Lemmas 2.3 and 2.2 to conclude that $\mathcal{P}_{1}=\mathcal{P}_{2}$.

Corollary 3.6. For a nilpotent group $G$ the following statements are equivalent:

(1) $\mathbf{Z} / p^{\infty} \notin \sigma(G)$,

(2) $\widetilde{H}_{*}\left(G ; \mathbf{Z} / p^{\infty}\right)=0$, and

(3) $H_{1}\left(G ; \mathbf{Z} / p^{\infty}\right)=H_{2}\left(G ; \mathbf{Z} / p^{\infty}\right)=0$.

Proof. If $\mathbf{Z} / p^{\infty} \notin \sigma(G)$, then $G$ is $\bar{p}$-local, so all its integral homology groups are $\bar{p}$ local and $H_{i}\left(G ; \mathbf{Z} / p^{\infty}\right)=0$ for all $i \geq 1$, which proves the implication (1) $\Longrightarrow(2)$.

Notice $(2) \Longleftrightarrow(3)$ by Corollary 2.4. Indeed, if $A$ is Abelian and $H_{1}\left(A ; \mathbf{Z} / p^{\infty}\right)=$ $H_{2}\left(A ; \mathbf{Z} / p^{\infty}\right)=0$, then $A$ must be $\bar{p}$-local.

Let $\mathcal{P}_{1}$ be the class of $\bar{p}$-local nilpotent groups and let $\mathcal{P}_{2}$ be the class of nilpotent groups such that $H_{1}\left(G ; \mathbf{Z} / p^{\infty}\right)=H_{2}\left(G ; \mathbf{Z} / p^{\infty}\right)=0$. Use Lemma 2.3 to conclude that $\mathcal{P}_{1}=\mathcal{P}_{2}$.

Corollary 3.7. For a nilpotent group $G$ the following statements are equivalent:

(1) $\mathbf{Z}_{(p)} \notin \sigma(G)$,

(2) $\widetilde{H}_{*}\left(F(G) ; \mathbf{Z} / p^{\infty}\right)=0$, and

(3) $H_{1}\left(F(G) ; \mathbf{Z} / p^{\infty}\right)=H_{2}\left(F(G) ; \mathbf{Z} / p^{\infty}\right)=0$.

It is obvious that $\mathbf{Z} / p^{\infty} \notin \sigma(G)$ if and only if $G \rightarrow G_{(\bar{p})}$ is an isomorphism. The following lemma characterizes $\mathbf{Z}_{(p)} \notin \sigma(G)$ via localizations.

Lemma 3.8. For a nilpotent group $G$ the following statements are equivalent:

(1) $\mathbf{Z}_{(p)} \notin \sigma(G)$,

(2) $G \rightarrow G_{(\bar{p})}$ is an epimorphism.

Proof. If $G \rightarrow G_{(\bar{p})}$ is an epimorphism, then its kernel has trivial $\bar{p}$-localization (by exactness of the localization functor) and must be a torsion group. Therefore $F(G)=F\left(G_{(\bar{p})}\right)$ is $\bar{p}$-local. If $F(G)$ is $\bar{p}$-local, then apply exactness of the localization functor to the short exact sequence $1 \rightarrow \operatorname{Tor}(G) \rightarrow G \rightarrow F(G) \rightarrow 1$ and derive that $G \rightarrow G_{(\bar{p})}$ is an epimorphism.

Definition 3.9. The torsion-divisible Bockstein basis $\sigma_{T D}(G)$ of $G$ consists of all $\mathbf{Z} / p^{\infty}$ belonging to $\sigma(G)$. We set $\sigma_{N T D}(G)=\sigma(G) \backslash \sigma_{T D}(G)$.

Lemma 3.10. If $G \rightarrow I$ is an epimorphism of nilpotent groups. Then $\sigma_{N T D}(I) \subset$ $\sigma_{N T D}(G)$. 
Proof. Suppose $\mathbf{Q} \notin \sigma(G)$. Then $G$ is a torsion group. So $I$ is a torsion group; hence $\mathbf{Q} \notin \sigma(I)$.

Let $\mathbf{Z} / p \notin \sigma(G)$. Then $G$ is $p$-divisible and then also $I$ is $p$-divisible.

Let $\mathbf{Z}_{(p)} \notin \sigma(G)$. Then $G \rightarrow G_{(\bar{p})}$ is an epimorphism. Because $\bar{p}$-localization is an exact functor, the map $G_{(\bar{p})} \rightarrow I_{(\bar{p})}$ is an epimorphism and hence $I \rightarrow I_{(\bar{p})}$ is an epimorphism.

Lemma 3.11. Let $1 \rightarrow K \rightarrow G \rightarrow I \rightarrow 1$ be a central extension of nilpotent groups. Then $\sigma(G) \subset \sigma(K) \cup \sigma(I)$.

Proof. Let $\mathbf{Q} \notin \sigma(K) \cup \sigma(I)$. Then $K$ and $I$ are torsion groups. Hence $G$ is torsion, so $\mathbf{Q} \notin \sigma(G)$.

Let $\mathbf{Z} / p \notin \sigma(K) \cup \sigma(I)$. Then $K$ and $I$ are $p$-divisible. By Lemma 2.2 also $G$ is $p$-divisible.

Let $\mathbf{Z} / p^{\infty} \notin \sigma(K) \cup \sigma(I)$. Then $K \rightarrow K_{(\bar{p})}$ and $I \rightarrow I_{(\bar{p})}$ are isomorphisms. Using the Five Lemma and the fact that $\bar{p}$-localization is an exact functor, we conclude that also $G \rightarrow G_{(\bar{p})}$ is an isomorphism.

Let $\mathbf{Z}_{(p)} \notin \sigma(K) \cup \sigma(I)$. Then $K \rightarrow K_{(\bar{p})}$ and $I \rightarrow I_{(\bar{p})}$ are epimorphisms. By the Three Lemma [10, Lemma 2.8], also $G \rightarrow G_{(\bar{p})}$ is an epimorphism.

Lemma 3.12. Let $1 \rightarrow K \rightarrow G \rightarrow I \rightarrow 1$ be a nilpotent central extension of groups. If $\mathbf{Z}_{(p)} \notin \sigma(A b(G))$ for some prime $p$, then $\mathbf{Z}_{(p)} \notin \sigma(K)$ and $\mathbf{Z}_{(p)} \notin \sigma(A b(I))$.

Proof. Assume $\mathbf{Z}_{(p)} \notin \sigma(\operatorname{Ab}(G))$. That means the map $F(\operatorname{Ab}(G)) \rightarrow F(\operatorname{Ab}(G))_{(\bar{p})}$ is an isomorphism. Because $\operatorname{Ab}(G) \rightarrow \mathrm{Ab}(I)$ is an epimorphism and $F$ is a right exact functor, the map $F(\operatorname{Ab}(G)) \rightarrow F(\mathrm{Ab}(I))$ is an epimorphism. Hence the map $F(\operatorname{Ab}(I)) \rightarrow F(\operatorname{Ab}(I))_{(\bar{p})}$ is an epimorphism. Its kernel is a $p$-torsion group, so the kernel is trivial and the map $F(\mathrm{Ab}(I)) \rightarrow F(\mathrm{Ab}(I))_{(\bar{p})}$ is an isomorphism. That means $\mathbf{Z}_{(p)} \notin \sigma(\mathrm{Ab}(I))$.

There exists an epimorphism $\otimes^{n} \mathrm{Ab}(G) \rightarrow K$ for some integer $n$. Because $F\left(\mathrm{Ab}\left(\otimes^{n} G\right)\right) \rightarrow F\left(\operatorname{Ab}\left(\otimes^{n} G\right)\right)_{(\bar{p})}$ is an isomorphism in the same way as in the previous paragraph, we can prove that $F(K) \rightarrow F(K)_{(\bar{p})}$ is an isomorphism. Hence $\mathbf{Z}_{(p)} \notin \sigma(K)$.

Lemma 3.13. Let $G$ be a nilpotent group. Then $\sigma_{N T D}(G)=\sigma_{N T D}(A b(G))$ and $\sigma(A b(G)) \subset \sigma(G)$.

Proof. The inclusion $\sigma_{N T D}(\operatorname{Ab}(G)) \subset \sigma_{N T D}(G)$ follows from Lemma 3.10 .

Let us prove $\sigma_{N T D}(G) \subset \sigma_{N T D}(\operatorname{Ab}(G))$. Suppose $\mathbf{Q} \notin \sigma_{N T D}(\operatorname{Ab}(G))$. Because $G$ is a torsion group if and only if $\mathrm{Ab}(G)$ is a torsion group, $\mathbf{Q} \notin \sigma_{N T D}(G)$.

Suppose $\mathbf{Z} / p \notin \sigma_{N T D}(\operatorname{Ab}(G))$. Because $G$ is $p$-divisible if and only if $\operatorname{Ab}(G)$ is $p$-divisible, $\mathbf{Z} / p \notin \sigma_{N T D}(G)$.

Consider the class $\mathcal{P}$ of all nilpotent groups such that $\mathbf{Z}_{(p)} \notin \sigma_{N T D}(\operatorname{Ab}(G))$ implies $\mathbf{Z}_{(p)} \notin \sigma_{N T D}(G)$. $\mathcal{P}$ clearly contains all Abelian groups. To show $\mathcal{P}$ equals the class $\mathcal{N}$ of all nilpotent groups it suffices to show (see Lemma 2.3) that for any nilpotent central extension $A \rightarrow G \rightarrow G^{\prime}$ such that the Hirsch length of $G^{\prime}$ is less than $h(G), A, G^{\prime} \in \mathcal{P}$ implies $G \in \mathcal{P}$. Assume $\mathbf{Z}_{(p)} \notin \sigma_{N T D}(\operatorname{Ab}(G))$. By Lemma 3.12, we conclude $\mathbf{Z}_{(p)} \notin \sigma_{N T D}\left(G^{\prime}\right)$ as $G^{\prime} \in \mathcal{P}$ and $\mathbf{Z}_{(p)} \notin \sigma_{N T D}(A)$. By Lemma 3.11, $\mathbf{Z}_{(p)} \notin \sigma_{N T D}(G)$.

Let us now prove that $\sigma(\operatorname{Ab}(G)) \subset \sigma(G)$. By Lemma 3.10, $\sigma_{N T D}(\operatorname{Ab}(G)) \subset$ $\sigma_{N T D}(G)$. Suppose $\mathbf{Z} / p^{\infty} \notin \sigma(G)$. Then $G$ is uniquely $p$-divisible, so $H_{*}(G ; \mathbf{Z})$ is 
uniquely $p$-divisible. In particular $\operatorname{Ab}(G)=H_{1}(G ; \mathbf{Z})$ is uniquely $p$-divisible; hence $\mathbf{Z} / p^{\infty} \notin \sigma(\operatorname{Ab}(G))$.

Theorem 3.14. If $1 \rightarrow K \rightarrow G \rightarrow I \rightarrow 1$ is a nilpotent central extension, then $\sigma(G)=\sigma(K) \cup \sigma(I)$.

Proof. By Lemma 3.11 $\sigma(G) \subset \sigma(K) \cup \sigma(I)$.

Let us prove that $\sigma(K) \cup \sigma(I) \subset \sigma(G)$. Suppose $\mathbf{Q} \notin \sigma(G)$. Then $G$ is a torsion group. Therefore $K$ and $I$ are also torsion groups, so $\mathbf{Q} \notin \sigma(K) \cup \sigma(I)$.

Suppose $\mathbf{Z} / p \notin \sigma(G)$. Then $G$ is $p$-divisible. By Lemma 2.2, $K$ and $I$ are $p$-divisible, so $\mathbf{Z} / p \notin \sigma(K) \cup \sigma(I)$.

Suppose $\mathbf{Z} / p^{\infty} \notin \sigma(G)$. Then $G \rightarrow G_{(\bar{p})}$ is an isomorphism. Because $\bar{p}$ localization is an exact functor, the map $K \rightarrow K_{(\bar{p})}$ is a monomorphism and the map $\operatorname{Ab}(G) \rightarrow(\operatorname{Ab}(G))_{(\bar{p})}$ is an epimorphism. Because there exists an epimorphism $\otimes^{n} \mathrm{AbG} \rightarrow K$, the map $K \rightarrow K_{(\bar{p})}$ is also an epimorphism; hence it is an isomorphism. By the Five Lemma, also the map $I \rightarrow I_{(\bar{p})}$ is an isomorphism, so $\mathbf{Z} / p^{\infty} \notin \sigma(K) \cup \sigma(I)$.

If $\mathbf{Z}_{(p)} \notin \sigma(G)$, then $\mathbf{Z}_{(p)} \notin \sigma(K)$ and $\mathbf{Z}_{(p)} \notin \sigma(I)$ by Lemmas 3.12 and 3.13 ,

\section{Bockstein SPACES}

Definition 4.1. A metrizable space $X$ is called a Bockstein space if $\operatorname{dim}_{G} X=$ $\sup \left\{\operatorname{dim}_{H} X \mid H \in \sigma(G)\right\}$ for all Abelian groups $G$.

Remark 4.2. In the above definition observe that $\operatorname{dim}_{G} X$ is an element of $\mathbf{N} \cup\{0, \infty\}$ and not only in $\{0,1, \infty\}$ as in the case of non-Abelian groups $G$.

Dranishnikov-Repovš-Shchepin [6] showed the existence of a separable metric space $X$ of dimension 2 such that $\operatorname{dim}_{\mathbf{Z}_{(p)}} X=1$ for all primes $p$. Thus, $X$ is not a Bockstein space as $\operatorname{dim}_{\mathbf{Z}} X=2>1=\sup \left\{\operatorname{dim}_{H} X \mid H \in \sigma(\mathbf{Z})\right\}$.

Problem 4.3. Is every metric ANR a Bockstein space?

Proposition 4.4. Suppose $X=\bigcup_{n=1}^{\infty} X_{n}$ is metrizable and each $X_{n}$ is closed in $X$. If all $X_{n}$ are Bockstein spaces, then so is $X$.

Proof. Suppose $G$ is an Abelian group and $H \in \sigma(G)$. If $\operatorname{dim}_{G}(X) \leq m$, then $\operatorname{dim}_{G}\left(X_{n}\right) \leq m$ for all $n$ and $\operatorname{dim}_{H}\left(X_{n}\right) \leq m$ for all $n$ resulting in $\operatorname{dim}_{H}(X) \leq m$.

If $\operatorname{dim}_{H}(X)<m$ for all $H \in \sigma(G)$, then $\operatorname{dim}_{H}\left(X_{n}\right)<m$ for all $n$ and $\operatorname{dim}_{G}\left(X_{n}\right)$ $<m$ for all $n$ resulting in $\operatorname{dim}_{G}(X)<m$.

For a subset $l \subset \mathbb{P}$ of prime integers let $\mathbf{Z}_{l}=\left\{\frac{m}{n} \in \mathbf{Q} \mid n\right.$ is not divisible by any $p \in l\}$ and let $\hat{\mathbf{Z}}_{l}$ be the group of $l$-adic integers.

Theorem 4.5. A metrizable space $X$ is a Bockstein space if and only if $\operatorname{dim}_{\mathbf{Z}_{l}} X=$ $\operatorname{dim}_{\hat{\mathbf{z}}_{l}} X$ for all subsets $l \subset \mathbb{P}$ of the set of prime numbers.

Proof. Since $\sigma\left(\hat{\mathbf{Z}}_{l}\right)=\sigma\left(\mathbf{Z}_{l}\right)$ for all $l \subset \mathbb{P}, \operatorname{dim}_{\mathbf{Z}_{l}} X=\operatorname{dim}_{\hat{\mathbf{Z}}_{l}} X$ holds for any Bockstein space $X$. 
Assume $\operatorname{dim}_{\mathbf{Z}_{l}} X=\operatorname{dim}_{\hat{\mathbf{z}}_{l}} X$ for all subsets $l \subset \mathbb{P}$ of the set of prime numbers. Suppose $G$ is a torsion-free Abelian group $G$. If $\mathbf{Z}_{(p)} \in \sigma(G)$, then Theorem B(d) of [7] says that $\operatorname{dim}_{\hat{\mathbf{z}}_{(p)}} X \leq \operatorname{dim}_{G} X$. Therefore $\operatorname{dim}_{G} X \geq \sup \left\{\operatorname{dim}_{H} X \mid H \in\right.$ $\sigma(G)\}$. Suppose $\sup \left\{\operatorname{dim}_{H} X \mid H \in \sigma(G)\right\}=n$ and consider $l=\{p \mid p \cdot G \neq$ $G$ \}. Theorem B(f) of [7] says that $\operatorname{dim}_{G}(X) \leq \operatorname{dim}_{\mathbf{Z}_{l}} X$. Since $\sigma(G)=\sigma\left(\mathbf{Z}_{l}\right)$, $\operatorname{dim}_{G} X \leq \operatorname{dim}_{\mathbf{Z}_{l}} X=\sup \left\{\operatorname{dim}_{H} X \mid H \in \sigma\left(\mathbf{Z}_{l}\right)\right\}=\sup \left\{\operatorname{dim}_{H} X \mid H \in \sigma(G)\right\}=n$. That proves $\operatorname{dim}_{G} X=\sup \left\{\operatorname{dim}_{H} X \mid H \in \sigma(G)\right\}$ for all torsion-free Abelian groups. The same equality holds for all torsion Abelian groups by Theorem B(a) of [7. In the case of arbitrary Abelian groups $G$, as $\sigma(G)=\sigma(F(G)) \cup \sigma(\operatorname{Tor}(G))$ and $\operatorname{dim}_{G}=\max \left(\operatorname{dim}_{F(G)} X, \operatorname{dim}_{\text {Tor }(G)} X\right)\left(\right.$ see Theorem B(b) of [7]) one gets $\operatorname{dim}_{G} X=$ $\sup \left\{\operatorname{dim}_{H} X \mid H \in \sigma(G)\right\}$ as well.

Remark 4.6. Notice that it is not sufficient to assume $\operatorname{dim}_{\mathbf{Z}_{(p)}} X=\operatorname{dim}_{\hat{\mathbf{Z}}_{(p)}} X$ for all primes $p$ in Theorem 4.5] Indeed, the space $X$ in [6] has that property as $1=\operatorname{dim}_{\mathbf{z}_{(p)}} X \geq \operatorname{dim}_{\hat{\mathbf{z}}_{(p)}} X \geq 1$ for all primes $p$.

Theorem 4.7. Let $X$ be a Bockstein space. If $G$ is nilpotent, then $\operatorname{dim}_{G}(X) \leq 1$ if and only if $\sup \left\{\operatorname{dim}_{H}(X) \mid H \in \sigma(G)\right\} \leq 1$.

Proof. Let $\mathcal{P}_{1}$ be the class of all nilpotent groups and let $\mathcal{P}_{2}$ be the class of nilpotent groups $G$ such that $\operatorname{dim}_{G}(X) \leq 1$ if and only if $\sup \left\{\operatorname{dim}_{H}(X) \mid H \in \sigma(G)\right\} \leq 1$. Since $\mathcal{P}_{2}$ contains all Abelian groups, in view of Lemma 2.3 it suffices to show that for any nilpotent central extension $K \rightarrow G \rightarrow I$ the conditions $K, I \in \mathcal{P}_{2}$ imply $G \in \mathcal{P}_{2}$. It is so if $G$ is Abelian, so assume $G$ is not Abelian. Moreover, as $\sigma(\operatorname{Ab}(G)) \subset \sigma(G)$ by Lemma 3.13 and $\operatorname{dim}_{G} X \leq 1$ implies $\operatorname{dim}_{\mathrm{Ab}(G)} X \leq 1$ (see Lemma 1.4), either $\operatorname{dim}_{G}(X) \leq 1$ or sup $\left\{\operatorname{dim}_{H}(X) \mid H \in \sigma(G)\right\} \leq 1$ implies $\operatorname{dim}_{\mathrm{Ab}(G)} X \leq 1$, so we may as well assume $\operatorname{dim}_{\mathrm{Ab}(G)} X \leq 1$.

In view of Lemma 1.2 and the fact that $\operatorname{Ab}(G)=1$ implies $G=1$, the equivalence of conditions $\operatorname{dim}_{G}(X) \leq 1$ and $\sup \left\{\operatorname{dim}_{H}(X) \mid H \in \sigma(G)\right\} \leq 1$ may fail only if $\operatorname{dim}_{\mathrm{Ab}(G)} X=1$, so assume $\operatorname{dim}_{\mathrm{Ab}(G)} X=1$.

Suppose $\operatorname{dim}_{H}(X)=n>1$ for some $H \in \sigma(K)$. If $H \neq \mathbf{Z} / p^{\infty}$, then $H \in$ $\sigma_{N T D}(G)=\sigma_{N T D}(\operatorname{Ab}(G))$ (Theorem 3.14 and Lemma 3.13), a contradiction. So $H=\mathbf{Z} / p^{\infty}$ for some prime $p$ and $\mathbf{Z} / p^{\infty} \notin \sigma(\operatorname{Ab}(G))$. Hence $\operatorname{Ab}(G)$ is $p$-divisible and this is equivalent to $G$ being $p$-divisible [2, Lemma 5.1]. Because $\mathbf{Z} / p^{\infty} \notin$ $\sigma(\operatorname{Ab}(G))$, by Lemma 3.6 $H_{1}\left(\operatorname{Ab}(G) ; \mathbf{Z} / p^{\infty}\right)=0$, so also $H_{1}\left(G ; \mathbf{Z} / p^{\infty}\right)=0$. By Lemma 3.6. $H_{2}\left(G ; \mathbf{Z} / p^{\infty}\right) \neq 0$ as $\mathbf{Z} / p^{\infty} \in \sigma(G)$. This implies that $F\left(H_{2}(G ; \mathbf{Z})\right)$ is not $p$-divisible, so $\mathbf{Z}_{(p)} \in \sigma\left(H_{2}(G ; \mathbf{Z})\right)$. If $G$ is not a torsion group, then $\operatorname{Ab}(G)$ is not a torsion group; hence by definition $\mathbf{Q} \in \sigma(\operatorname{Ab}(G))$. Therefore $\operatorname{dim}_{\mathbf{Q}}(X) \leq 1$. Using that fact and the Bockstein Inequalities (BI5, BI6 [12]), we get $\operatorname{dim}_{\mathbf{Z} / p^{\infty}}(X)=\operatorname{dim}_{\mathbf{Z}_{(p)}}(X)-1$. Because $\mathbf{Z}_{(p)} \in \sigma\left(H_{2}(G ; \mathbf{Z})\right)$, the dimension $\operatorname{dim}_{\mathbf{Z}_{(p)}}(X) \leq \operatorname{dim}_{H_{2}(G ; \mathbf{Z})}(X) \leq 2$ as $X$ is a Bockstein space and then $\operatorname{dim}_{\mathbf{Z} / p^{\infty}}(X)=\operatorname{dim}_{\mathbf{Z}_{(p)}}(X)-1 \leq 1$, a contradiction.

Thus $G$ is a torsion group and is a product of $q$-groups $G=\prod_{q \in \mathbb{P}} G_{q}$. Hence $\operatorname{Ab}(G)=\prod_{q \in \mathbb{P}} \operatorname{Ab}\left(G_{q}\right)$. Because $G$ is not $\bar{p}$-local, $G_{p} \neq 1$, but $\operatorname{Ab}(G)$ is uniquely $p$-divisible, so $\operatorname{Ab}\left(G_{p}\right)=1$. Therefore $G_{p}$ is a perfect nilpotent group, but such a group is trivial, a contradiction.

Thus $\operatorname{dim}_{H} X \leq 1$ for all $H \in \sigma(K)$ and $\operatorname{dim}_{K} X=\sup \left\{\operatorname{dim}_{H}(X) \mid H \in \sigma(K)\right\} \leq$ 1 as $K$ is Abelian and $X$ is a Bockstein space. 
Suppose $\sup \left\{\operatorname{dim}_{H}(X) \mid H \in \sigma(G)\right\} \leq 1$. By Theorem 3.14 $\sigma(G)=\sigma(K) \cup \sigma(I)$. Therefore $\sup \left\{\operatorname{dim}_{H}(X) \mid H \in \sigma(I)\right\} \leq 1$ and $\operatorname{dim}_{I} X \leq 1$ as $I \in \mathcal{P}_{2}$. Consequently, $\operatorname{dim}_{G} X \leq 1$ by Lemma 1.3 .

Suppose $\operatorname{dim}_{G} X \leq 1$. By Lemma 1.3 one gets $\operatorname{dim}_{I} X \leq 1$ and $\sup \left\{\operatorname{dim}_{H}(X) \mid H \in\right.$ $\sigma(I)\} \leq 1$ as $I \in \mathcal{P}_{2}$. By Theorem 3.14. $\sigma(G)=\sigma(K) \cup \sigma(I)$; hence

$$
\sup \left\{\operatorname{dim}_{H}(X) \mid H \in \sigma(G)\right\}=\sup \left\{\operatorname{dim}_{H}(X) \mid H \in \sigma(K) \cup \sigma(I)\right\} \leq 1 .
$$

Corollary 4.8. Let $L$ be a connected nilpotent $C W$ complex. If $X$ is a Bockstein space and $\operatorname{dim}_{H_{n}(L)} X \leq n$ for all $n \geq 1$, then $\operatorname{dim}_{\pi_{n}(L)} X \leq n$ for all $n \geq 1$.

Proof. It is shown in 2] that $\operatorname{dim}_{\pi_{n}(L)} X \leq n$ for all $n \geq 2$, so it suffices to prove $\operatorname{dim}_{\pi_{1}(L)} X \leq 1$. If that inequality is false, then there is $H \in \sigma\left(\pi_{1}(L)\right)$ such that $\operatorname{dim}_{H}(X)>1$ (see Theorem 4.7). In view of Lemma 3.13, as $\operatorname{dim}_{H_{1}(L)}(X) \leq 1, H=$ $\mathbf{Z} / p^{\infty}$ for some prime $p$. Also, $H_{1}(L)$ is not a torsion group, so $\operatorname{dim}_{\mathbf{Q}}(X) \leq 1$. Using Bockstein Inequalities one gets $\operatorname{dim}_{\mathbf{z}_{(p)}}(X) \geq 3$. Therefore the $i$-th homology groups of both $L$ and $\tilde{L}$ with coefficients in $\mathbf{Z} / p^{\infty}$ vanish for $i \leq 2$. From the fibration $\tilde{L} \rightarrow L \rightarrow K\left(\pi_{1}(L), 1\right)$ one gets that the $i$-th homology groups of $K\left(\pi_{1}(L), 1\right)$ with coefficients in $\mathbf{Z} / p^{\infty}$ vanish for $i \leq 2$. However, in view of Corollary 3.6, that means $\mathbf{Z} / p^{\infty} \notin \sigma\left(\pi_{1}(L)\right)$, a contradiction.

Corollary 4.9. Let $L$ be a connected nilpotent $C W$ complex and let $X$ be a Bockstein space such that $\operatorname{dim}_{H_{n}(L)} X \leq n$ for all $n \geq 1$. If $X$ is finite dimensional or $X \in A N R$, then $X \tau L$.

Proof. By Corollary 4.8 one gets $\operatorname{dim}_{\pi_{n}(L)} X \leq n$ for all $n \geq 1$ and by Theorem $\mathrm{G}$ in [7] one has $X \tau L$.

\section{REFERENCES}

[1] K. S. Brown, Cohomology of groups. Graduate Texts in Mathematics, 87. Springer-Verlag, New York-Berlin, 1982. MR672956 (83k:20002)

[2] M. Cencelj, J. Dydak, A. Mitra, A. Vavpetič, Hurewicz-Serre Theorem in extension theory, Fundament. Math. 198 (2008), 2, 113-123. MR2369125 (2008k:54050)

[3] M. Cencelj, A. N. Dranishnikov, Extension of maps into nilpotent spaces. III, Topology Appl. 153 (2005), no. 2-3, 208-212. MR2175345 (2006j:55001)

[4] A. N. Dranishnikov, Cohomological dimension theory of compact metric spaces, Topology Atlas (1999).

[5] A. N. Dranishnikov, D. Repovš, Cohomological dimension with respect to perfect groups, Topology and its Applications 74 (1996), 123-140. MR1425932 (98e:55002)

[6] A. N. Dranishnikov, D. Repovš, E. Shchepin, Dimension of products with continua, Topology Proceedings 18 (1993), 57-73. MR.1305123 (96b:54054)

[7] J. Dydak, Cohomological dimension and metrizable spaces, Trans. Amer. Math. Soc. 337 (1993), 219-234. MR1153013 (93g:55001)

[8] J. Dydak, Cohomological dimension and metrizable spaces. II, Trans. Amer. Math. Soc. 348 (1996), 1647-1661. MR1333390 (96h:55001)

[9] J. Dydak, Extension dimension of paracompact spaces, Topology and its Applications 140 (2004), 227-243. MR2074918 (2005e:54033)

[10] P. Hilton, Nilpotente Gruppen und nilpotente Räume, Lecture Notes in Mathematics, Vol. 1053. Springer-Verlag, Berlin, 1984. MR764045 (86b:20001)

[11] P. Hilton, G. Mislin, J. Roitberg, Localization of nilpotent groups and spaces, North-Holland Publishing Co., Amsterdam-Oxford; American Elsevier Publishing Co., Inc., New York, 1975. MR $0478146(57: 17635)$

[12] V. I. Kuz'minov, Homological dimension theory, Russian Math. Surveys 23 (1968), 1-45. MR0240813 (39:2158) 
[13] D. J. S. Robinson, A course in the theory of groups, Springer-Verlag, New York, 1993. MR.1261639 (94m:20001)

[14] R. B. Warfield, Jr., Nilpotent groups, Lecture Notes in Mathematics, Vol. 513. SpringerVerlag, Berlin-New York, 1976. MR0409661 (53:13413)

Institute of Mathematics, Physics, and Mechanics, Jadranska ulica 19, SI-1111 LjublJANA, SLOVENIJA

E-mail address: matija.cencelj@guest.arnes.si

Department of Mathematics, University of Tennessee, Knoxville, Tennessee 37996

E-mail address: dydak@math.utk.edu

Department of Mathematics, University of Tennessee, Knoxville, Tennessee 37996

E-mail address: mitra@math.utk.edu

Current address: University of South Florida, 140 Seventh Avenue South, St. Petersburg, Florida 33701

E-mail address: atish.mitra@gmail.com

Fakulteta za Matematiko in Fiziko, Univerza v Ljubljani, Jadranska ulica 19, SI-1111 Ljubluana, Slovenija

E-mail address: ales.vavpetic@fmf.uni-lj.si 